\title{
Downregulation of long non-coding RNA ANRIL promotes proliferation and migration in hypoxic human pulmonary artery smooth muscle cells
}

\author{
SIQI WANG, CHEN ZHANG and XIAODAN ZHANG \\ College of Pharmacy, Harbin University of Commerce, Harbin, Heilongjiang 150076, P.R. China
}

Received May 23, 2019; Accepted September 26, 2019

DOI: $10.3892 / \mathrm{mmr} .2019 .10887$

\begin{abstract}
Pulmonary arterial hypertension (PAH) is a progressive syndrome. When PAH occurs, the circulatory resistance of the pulmonary vasculature will gradually increase, which may lead to right heart failure and death. Pathological features of PAH include abnormal proliferation of pulmonary vascular smooth muscle cells and pulmonary vascular remodeling. Hypoxia is the main cause of PAH, which directly induces the contraction and proliferation of pulmonary artery smooth muscle cells (PASMCs), and eventually leads to pulmonary vascular remodeling. Recent studies have shown that long non-coding RNAs (lncRNAs) play key roles in numerous biological processes, including cell proliferation and the occurrence and development of cardiovascular diseases. Studies have also shown that lncRNA antisense noncoding RNA in the INK4 locus (ANRIL) can promote the proliferation of vascular smooth muscle cells. Therefore, the hypothesis of the present study was that ANRIL may be expressed in PASMCs and play a regulatory role. In this study, the expression of ANRIL was analyzed by quantitative PCR. The effects of ANRIL on human pulmonary artery smooth muscle cells (HPASMCs) were assessed by MTT assay, flow cytometry, bromodeoxyuridine incorporation assay, Transwell assay, scratch-wound assay, immunofluorescence assay and western blotting. These experiments revealed that the expression of ANRIL was significantly downregulated in HPASMCs induced by hypoxia. The downregulation of ANRIL affected the cell cycle, making more HPASMCs move from the G0/G1 phase to the $\mathrm{G} 2 / \mathrm{M}+\mathrm{S}$ phase and strengthening the cell proliferation. Moreover, downregulated ANRIL increased the migration of HPASMCs under hypoxia. This study identified ANRIL as a critical regulator in HPASMCs
\end{abstract}

Correspondence to: Professor Xiaodan Zhang, College of Pharmacy, Harbin University of Commerce, 138 Tongda Street, Harbin, Heilongjiang 150076, P.R. China

E-mail: zhangxd85@163.com

Key words: pulmonary arterial hypertension, long non-coding RNAs, pulmonary artery smooth muscle cells, proliferation, migration induced by hypoxia and demonstrated the potential of gene therapy and drug development for treating PAH.

\section{Introduction}

Pulmonary arterial hypertension $(\mathrm{PAH})$ is an intractable cardiovascular disease, a key feature of which is pulmonary vascular remodeling (PVR) (1). Hypoxia is considered to be the predominant factor in the pathogenesis of PAH $(2,3)$. During the hypoxic exposure, the dysfunction of pulmonary artery smooth muscle cells (PASMCs), including both proliferation and migration, is the major reason for medial hypertrophy in PVR (4). To limit morbidity and mortality, attention has been focused on identifying the cellular and molecular mechanisms underlying aberrant proliferation and migration in human $(\mathrm{H})$ PASMCs. However, the mechanism of PASMCs proliferation and migration at the molecular level is still not very clear, and further research is needed.

Long non-coding RNAs (lncRNAs), with transcripts $>200$ nucleotides in length, were once considered as irrelevant transcriptional 'noise' without biological function. Previous studies, however, have shown that IncRNAs play a significant role in diverse biological and disease processes (5). LncRNAs can be used as a 'scaffold' to bind a variety of proteins to exert biological functions (6). Also, lncRNAs can form an anchor point to regulate different bioactivities by collecting or sequestering certain protein factors and participating in the synthesis and reconstruction of nucleic acid sequences (7).

On chromosome 9p21, there is a lncRNA named antisense noncoding RNA in the INK4 locus (ANRIL) with $\sim 126,000$ bps (8). LncRNA ANRIL was first identified and named in the investigation of a melanoma-neural system tumor syndrome family (9). A previous study identified that ANRIL is associated with $\sim 40 \%$ of all tumor types (10). Moreover, ANRIL also plays an important role in the development and progression of various diseases to a certain degree, including coronary heart disease $(11,12)$. Knocking down the expression of ANRIL in human aortic vascular smooth muscle cells, the results obtained suggested that ANRIL splicing variants played a role in coordinating tissue remodeling (13). Based on the above evidence, it is possible to hypothesize that ANRIL may regulate the process of $\mathrm{PAH}$ and that this deserves further investigation. Based on the potential roles of ANRIL in maintaining cellular functions, the present study speculated 
that ANRIL participates in hypoxia-induced proliferation and migration of HPASMCs in PAH. To this end, the expression of ANRIL was studied in HPASMCs where PAH had been induced by hypoxia.

\section{Materials and methods}

Materials. Antibodies against proliferating cell nuclear antigen (PCNA), Cyclin A, Cyclin D and Cyclin E were purchased from Boster Biological Technology. Antibody against Ki67 was purchased from Abcam. Bromodeoxyuridine (BrdU) proliferation assay kit was purchased from EMD Millipore. DMEM was purchased from Hyclone; GE Healthcare Life Sciences. The remaining chemical reagents were domestic analytical pure and biochemical reagents.

Cell culture. HPASMCs were obtained from the Center Laboratory of Harbin Medical University Daqing Campus. The cells were secondary cultured in 20\% Clark serum (CLARK Bioscience)-DMEM at $37^{\circ} \mathrm{C}$ in a $5 \% \mathrm{CO}_{2}$ humidified incubator. HPASMCs were cultured with a gas mixture containing $92 \%$ $\mathrm{N}_{2}, 5 \% \mathrm{CO}_{2}$ and $3 \% \mathrm{O}_{2}$ to create hypoxic conditions.

Small interfering (si)RNA and transfection. The sequence of siRNA against lncRNA ANRIL and scrambled negative control siRNA (si-NC) were synthesized by Shanghai GenePharma Co., Ltd. The target sequences of these siRNAs were follows: Si-IncRNA ANRIL, 5'-GCCCAAGCAUAUAGAUCAATT UUGAUCUAUAUGCUUGGGCTT-3' and si-NC, 5'-UUC UCCGAACGUGUCACGUTTACGUGACACGUUCGGAGA ATT-3'. HPASMCs cultured on six-well plates were transfected with the si-IncRNA ANRIL or si-NC (3.75 $\mu 1$ per $1.1 \mathrm{ml}$ DMEM) using X-tremeGENE (Sigma-Aldrich; Merck KGaA) (14). A total of $24 \mathrm{~h}$ post transfection, cells were harvested for quantitative (q)PCR and western blotting analyses.

Revers transcription- $q P C R$. Total RNA was extracted and isolated from HPASMCs using TRIzol reagent (Invitrogen; Thermo Fisher Scientific, Inc.). For the qPCR assay, the total RNA was reverse transcribed $\left(15 \mathrm{~min}\right.$ at $37^{\circ} \mathrm{C}, 5 \mathrm{~min}$ at $50^{\circ} \mathrm{C}$, 5 min at $98^{\circ} \mathrm{C}$ and holding at $4^{\circ} \mathrm{C}$.) into cDNA using One-step cDNA First Strand Reverse Transcription kit (HaiGene) according to the manufacturer's protocol. The expression levels were normalized against the expression of GAPDH. The PCR primers were as follows: ANRIL forward, 5'-TCC TGCCACTTCCTCTACTGA-3' and reverse, 5'-TGGTAT GGAAGGTGCTATGGA-3'; GAPDH forward, 5'-CAATGA CCCCTTCATTGACC-3' and reverse, 5'-TGGAAGATGGTG ATGGGATT-3'. The amplification reaction using $\mathrm{SYBR}^{\circledR}$ Green Realtime PCRMaster Mix was performed for $1 \mathrm{~min}$ at $95^{\circ} \mathrm{C}, 15 \mathrm{~min}$ at $95^{\circ} \mathrm{C}$, followed by 40 cycles at $95^{\circ} \mathrm{C}$ for $15 \mathrm{sec}$ and $52^{\circ} \mathrm{C}$ for $15 \mathrm{sec}$. The relative quantification of target genes expression was relative to the GAPDH levels. The $2^{-\Delta \Delta C q}$ method was used to calculate the expression of IncRNA ANRIL (15). All qPCR reactions were performed in duplicate.

Western blotting. Proteins were solubilized and extracted with lysis buffer (Tris $50 \mathrm{mM}, \mathrm{pH} 7.4, \mathrm{NaCl} 150 \mathrm{mM}$, Triton X-100 $1 \%$, EDTA $1 \mathrm{mM}$, and PMSF $2 \mathrm{mM}$ ) and incubated for $30 \mathrm{~min}$ on ice. The lysates were sonicated for $30 \mathrm{sec}$ at $25 \mathrm{kHz}$ in an ice bath and centrifuged at $15,000 \mathrm{x}$ g for $15 \mathrm{~min}$ at $4^{\circ} \mathrm{C}$ and the insoluble fractions were discarded. Protein concentrations were determined by the Bradford assay using bovine serum albumin (Sigma-Aldrich; Merck KGaA) as standard. Cell samples containing $20 \mu \mathrm{g}$ of protein were subjected to electrophoresis on a $10 \%$ SDS-polyacrylamide gel. Following electrophoresis, proteins were transferred to nitrocellulose membranes. These membranes were blocked at room temperature for $1 \mathrm{~h}$ in blocking buffer (Tris $20 \mathrm{mM}, \mathrm{pH}$ 7.6, $\mathrm{NaCl} 150 \mathrm{mM}$ and Tween 20 0.1\%) containing 5\% nonfat dry milk and incubated with PCNA (Boster Biological Technology; cat. no. BM3888; anti-PCNA antibody; 1:400), cyclin A (Boster Biological Technology; cat. no. BM4673; anti-Cyclin A2 antibody; 1:200), cyclin D (Boster Biological Technology; cat. no. BM4272; anti-Cyclin D1 antibody; 1:200), cyclin E (Boster Biological Technology; cat. no. BM4658; anti-Cyclin E2 antibody; 1:100), $\beta$-actin (Boster Biological Technology; cat. no. BM0627; anti- $\beta$-actin antibody; 1:2,000) at $4^{\circ} \mathrm{C}$ overnight. Secondary antibodies [Beyotime institute of Biotechnology; cat. no. A0208; horseradish peroxidase (HRP)-labeled Goat Anti-Rabbit $\operatorname{IgG}(\mathrm{H}+\mathrm{L}) ; 1: 10,000$; cat. no. A0216; HRP-labeled Goat Anti-Mouse $\operatorname{IgG}(\mathrm{H}+\mathrm{L})$; $1: 12,000]$ were prepared according to the corresponding proportion and species, and incubated at room temperature for $1 \mathrm{~h}$. The proteins were visualized with enhanced chemiluminescence reagents (Super Signal; Pierce; Thermo Fisher Scientific, Inc.). Grayscale values were calculated using Quantity One v4.6.2 (Bio-Rad Laboratories, Inc.) and statistical data were obtained using Microsoft Excel and Origin 7.5 (OriginLab).

Immunofluorescence assay. The pre-configured primary antibody against Ki67 (Santa Cruz Biotechnology, Inc.; cat. no. sc-23900, Ki-67 Antibody; 1:50) was added to the cells of different components and incubated at $4^{\circ} \mathrm{C}$ overnight. Then the cells were incubated with the secondary antibody [Invitrogen; Thermo Fisher Scientific, Inc.; cat. no. R-6393; Goat anti-Mouse IgG (H+L)] (1:100) conjugated with rhodamine for $1 \mathrm{~h}$ in the dark at $37^{\circ} \mathrm{C}$ and washed with PBS. The nuclei were stained with 4,6-diamidino-2-phenylindole (DAPI) at room temperature for $5 \mathrm{~min}$. The slides were examined using NA1.4 inverted fluorescence microscope (Leica DMI6000; Leica Microsystems, Inc.), images were visualized by Hamamatsu ORCA-R2 camera (Hamamatsu Photonics K.K.) and recorded by LAS AF 2.6.0 software (Leica Microsystems, Inc.). The experiments were conducted in triplicate.

Scratch-wound assay. The confluent HPASMCs cultured in 6-well plates were wounded by pipette tips and the detached cells were washed out by PBS (16). After that, the cells were treated with vehicle or the chemicals of interest in 5\% FBS-DMEM. The cells in all study groups were imaged in the same area of the culture plate immediately after $24 \mathrm{~h}$ after the wound was inflicted.

Transwell assay. Cell migration was measured using a Matrige $\mathrm{l}^{\circledR}$-coated modified Boyden chamber with a polycarbonate filter with a pore size of $8 \mu \mathrm{m}$. A total of $5 \times 10^{4}$ HPASMCs was added to each upper chamber in a serum-free medium. The lower chambers contained $20 \%$ Clark serum (CLARK Bioscience)-DMEM. After $24 \mathrm{~h}$ of incubation at $37^{\circ} \mathrm{C}$ 
in a $5 \% \mathrm{CO}_{2}$ humidified incubator, the nonmigrating cells in the upper chamber removed. The cells on the underside of the membrane were incubated in $4 \%$ formaldehyde solution for $10 \mathrm{~min}$ at room temperature, followed by incubation in $0.4 \%$ Crystal Violet for $5 \mathrm{~min}$ at room temperature. The number of migrated cells was measured by counting the number of stained nuclei per high power field using a light microscope (Olympus Corporation). Each sample was counted randomly at nine separate locations in the center of the membrane and HPASMCs migration activity was reported as the number of cells migrating per field of view. The experiments were performed at least three times in quadruplicate.

BrdU incorporation. HPASMCs were plated in 96-well plates at a density of $1 \times 10^{4}$ cells/well and then transfected by siRNA after growth arrest for $24 \mathrm{~h}$. BrdU was added to a final concentration of $30 \mu \mathrm{M}$ and incubated for $30-60 \mathrm{~min}$. BrdU was removed and washed 3 times with PBS. $4 \%$ paraformaldehyde was used to fix cells at room temperature for $30 \mathrm{~min}$. Then cells were washed three times with PBS. The following steps were done according to the manufacturer's protocol. Finally, the plate was read using a spectrophotometer microplate reader at dual wavelengths of $450 / 550 \mathrm{~nm}$.

3-(4,5-Dimethylthiazol-2-yl)-2,5-diphenyltetrazolium bromide (MTT) assay. After the model was established, the cells were drained of the original culture solution and $90 \mu \mathrm{M}$ culture solution and $10 \mu \mathrm{M} \mathrm{MTT}\left(5 \mathrm{mg} \cdot \mathrm{ml}^{-1}\right)$ were added to each well (17). The cultures were incubated at $37^{\circ} \mathrm{C}$ for $4 \mathrm{~h}$, then drained of liquid and $150 \mu 1 \mathrm{DMSO}$ was added to each well. The absorbance was read at $490 \mathrm{~nm}$ using a spectrophotometer, to determine the cell viability.

Cell cycle analysis. The proportions of cells in the G0/G1, S and $\mathrm{G} 2 / \mathrm{M}$ phases were detected using a flow cytometer. The cell cycle analysis kit (Beyotime institute of Biotechnology) was used. The cells were digested by trypsin then centrifuged at $1,000 \mathrm{x} g$ for $5 \mathrm{~min}$ at room temperature to remove the supernatant. A total of $1 \mathrm{ml}$ ice bath precooled PBS buffer was added to suspend the cells and centrifuged again (1 $000 \mathrm{x} \mathrm{g,} 5 \mathrm{~min}$, room temperature). A total of $1 \mathrm{ml} 70 \%$ ethanol precooled by ice bath was added and mixed. Cells were fixed at $4^{\circ} \mathrm{C}$ for $2 \mathrm{~h}$ or longer then centrifuged at 1,000 x $\mathrm{g}$ for 3-5 $\mathrm{min}$ at room temperature. A total of $1 \mathrm{ml}$ ice bath precooled PBS buffer was added then the mixture was centrifuged again $(1,000 \mathrm{x} \mathrm{g}$, 5 min, room temperature) and discard the supernatant. The prepared staining solution was added according to the protocol of the kit and the cell precipitation was resuspended. The cells were then placed in a temperature bath at $37^{\circ} \mathrm{C}$ for half an hour. Finally, the cells were filtered once through 400-mesh sieves and detected by flow cytometry (CytoFLEX, Beckman Coulter, Inc.). The results were analyzed by CytExpert 2.0 (Beckman Coulter, Inc.).

Statistical analysis. Each experiment was repeated $\geq 3$ times. The composite data were expressed as the mean \pm standard error of the mean. Statistical analysis was performed with analysis of variance followed by Dunnett's test or Student's t-test or Pearson correlation test. Quantity One-4.6.2 (Bio-Rad Laboratories, Inc.), Microsoft Office Excel 2007 (Microsoft

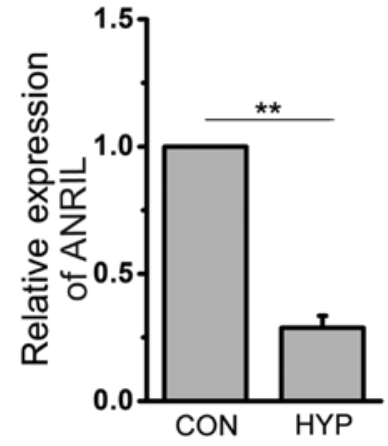

Figure 1. Quantitative PCR analysis of ANRIL expression in HPASMCs ${ }^{* *} \mathrm{P}<0.01(\mathrm{n}=6)$. CON, normoxia control; HYP, hypoxia; HPASMCs, human pulmonary artery smooth muscle cells; ANRIL, antisense noncoding RNA in the INK4 locus.
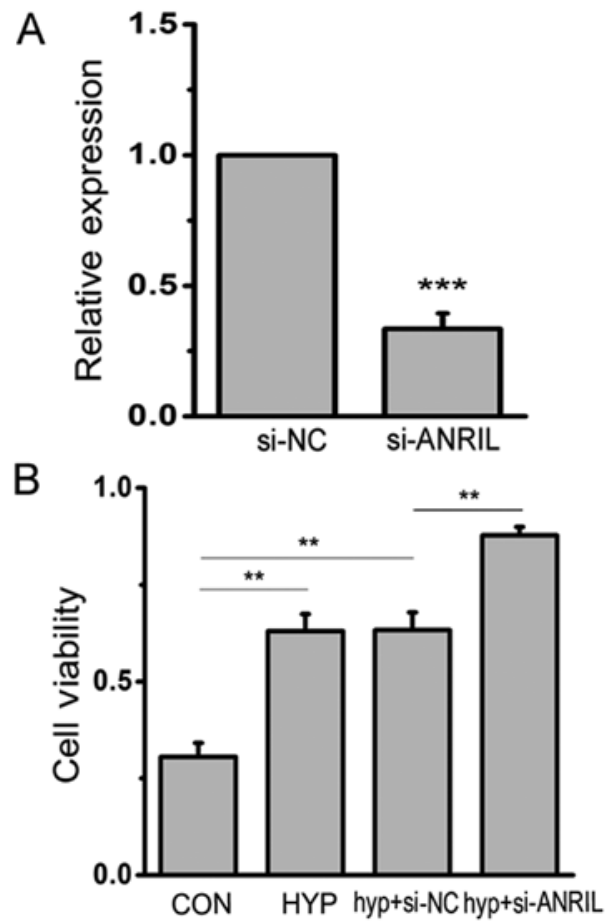

Figure 2. Downregulated ANRIL promotes the cell viability of HPASMCs in vitro. (A) ANRIL expression in HPASMCs following treatment with si-NC or si-ANRIL $(n=5)$. The experimental groups were established as follows: si-NC, cells treated with negative control; si-ANRIL, cells treated with si-ANRIL. (B) The MTT assay was used to detect the cell viability $(n=3)$. ${ }^{* *} \mathrm{P}<0.01$ and ${ }^{* * *} \mathrm{P}<0.001$. CON, normoxia control; HYP, hypoxia; hyp+si-NC, cells treated with the negative control under hypoxic conditions; hyp+si-ANRIL, cells treated with si-ANRIL under hypoxic conditions; HPASMCS, human pulmonary artery smooth muscle cells; si-ANRIL, small interfering RNA against antisense noncoding RNA in the INK4 locus.

Corporation), Origin 7.5 (OriginLab) and IPP 6.0 (Media Cybernetics, Inc.) software were used to analyze data. $\mathrm{P}<0.05$ was considered to indicate a statistically significant difference.

\section{Results}

ANRIL expression is downregulated in hypoxic HPASMCs. In order to prove that IncRNA ANRIL was related to hypoxic PAH, the expression of ANRIL in HPASMCs exposed to both normal and hypoxic conditions was detected by qPCR. The 

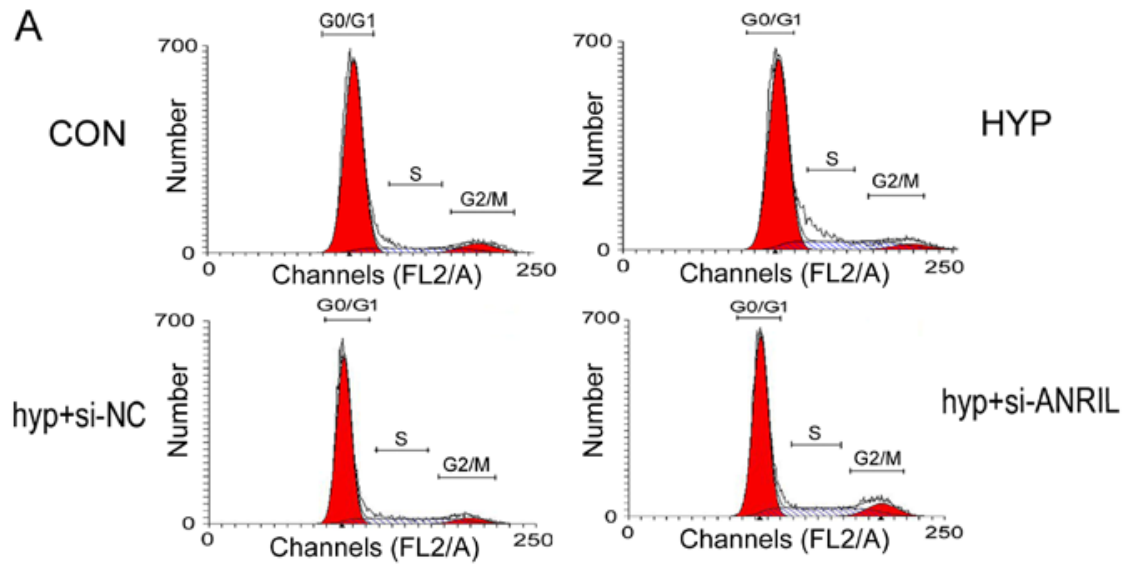

B

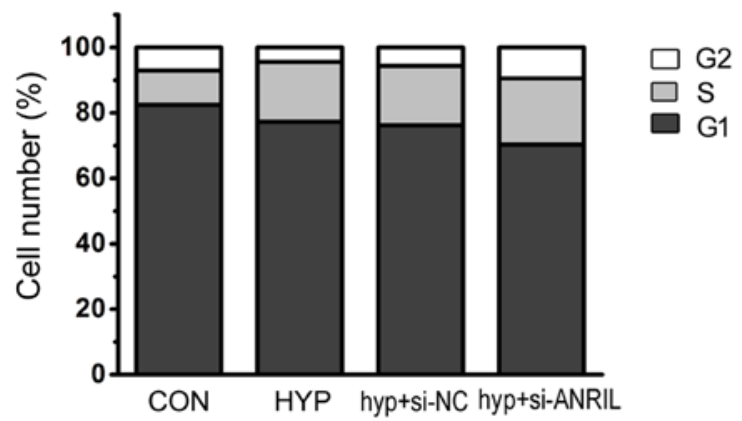

Figure 3. si-ANRIL induces cell cycle progression. ANRIL inhibited HPASMCs cycle progression and decreased the percentage of cells in the G2/M+S phase. (A) Hypoxia increased the cell number in the G2/M+S phases compared with normoxia, which was strengthened by si-ANRIL, as determined by flow cytometry analysis. (B) The percentage of cells in the S and G2/M phases in HPASMCs is shown. CON, normoxia control; HYP, hypoxia; hyp+si-NC, cells treated with negative control under hypoxic conditions; hyp+si-ANRIL, cells treated with si-ANRIL under hypoxic conditions (n=3); HPASMCS, human pulmonary artery smooth muscle cells; ANRIL, antisense noncoding RNA in the INK4 locus; si, small interfering.
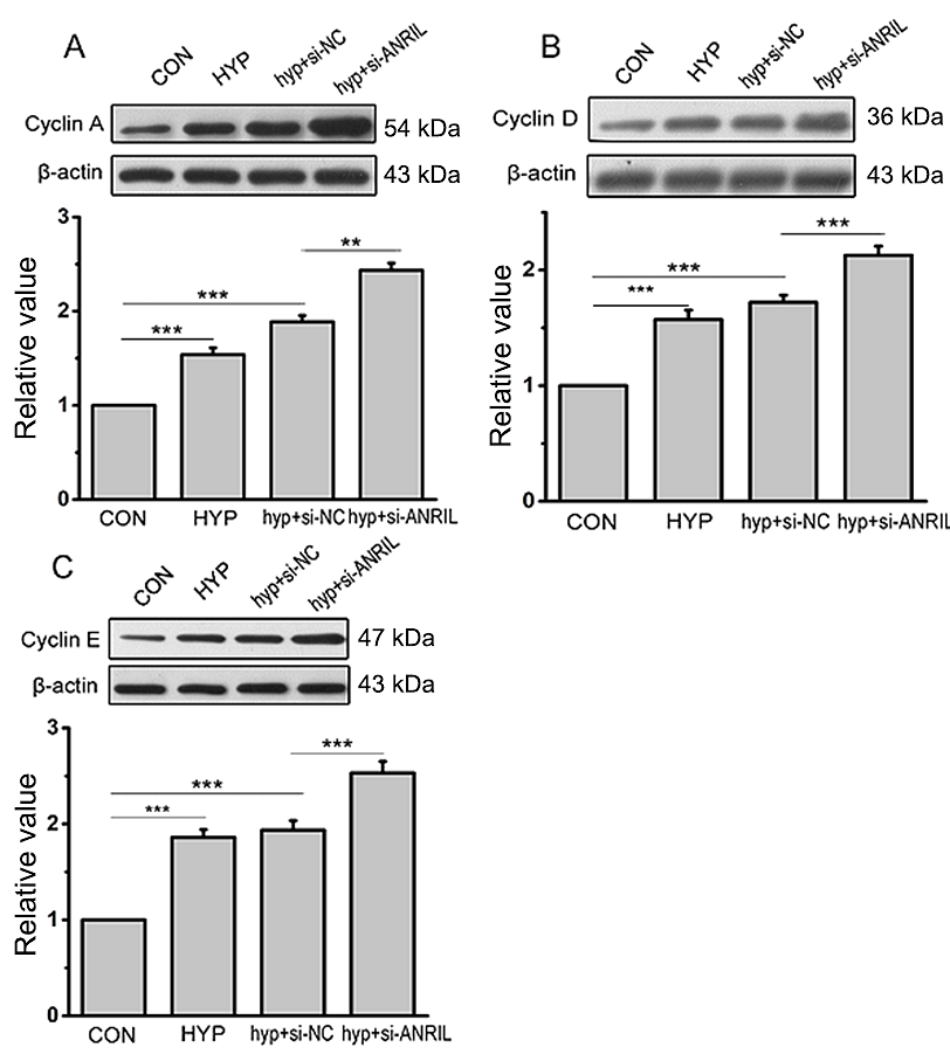

Figure 4. ANRIL regulates the expression levels of Cyclin A, Cyclin D and Cyclin E in HPASMCs under hypoxic conditions. (A) Cyclin A, (B) Cyclin D and (C) Cyclin E were analyzed by western blotting in different groups. ${ }^{* *} \mathrm{P}<0.01$ and ${ }^{* * * *} \mathrm{P}<0.001(\mathrm{n}=3)$. CON, normoxia control; HYP, hypoxia; hyp+Si-NC, cells treated with negative control under hypoxic conditions; hyp+si-ANRIL, cells treated with si-ANRIL under hypoxic conditions; HPASMCS, human pulmonary artery smooth muscle cells. 


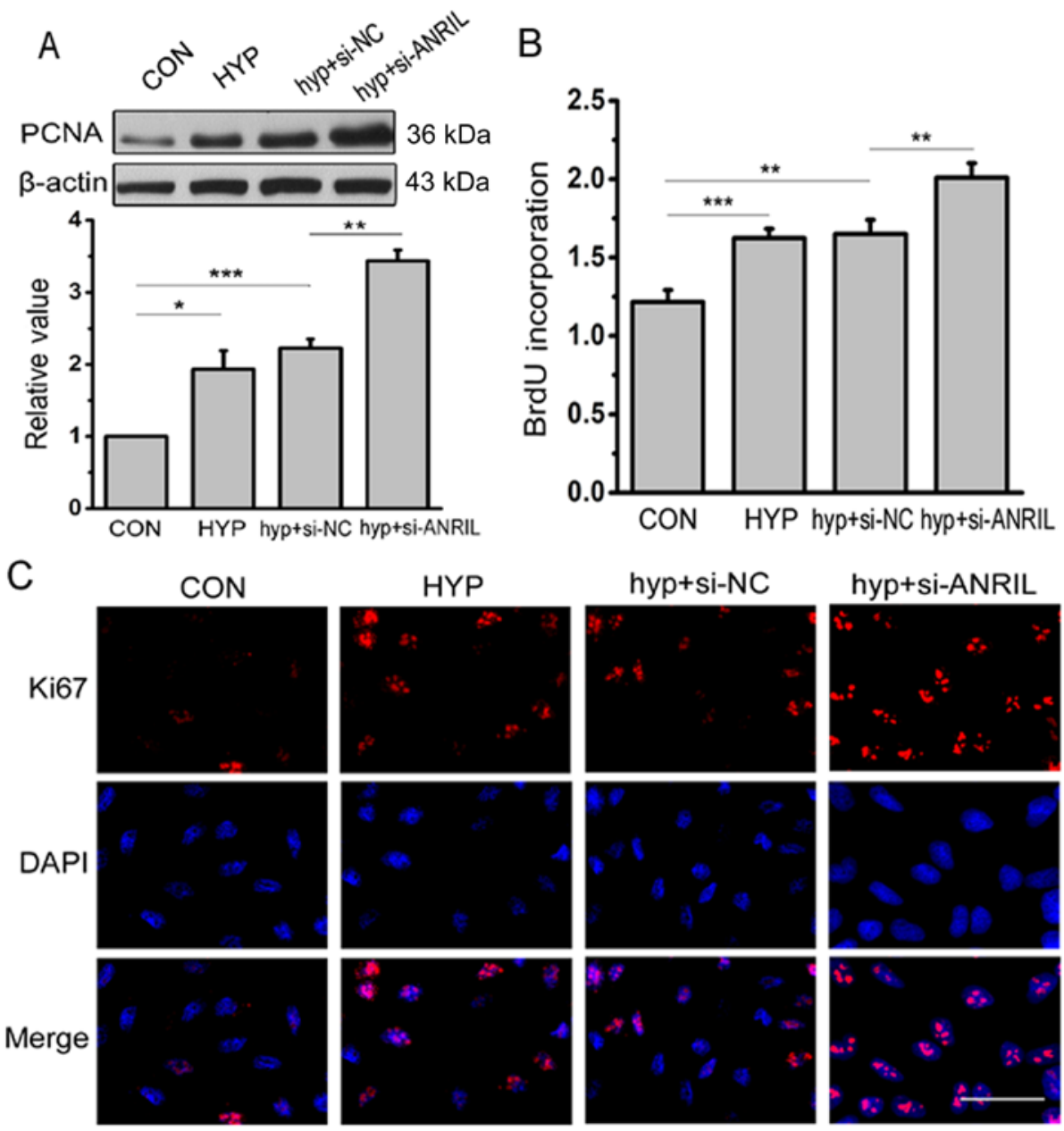

Figure 5. ANRIL induces higher levels of proliferation in HPASMCs. (A) The protein level of PCNA was analyzed by western blotting after transfection with si-ANRIL; densitometric quantification of protein bands showed a significant increase in PCNA following $24 \mathrm{~h}$ of transfection with si-ANRIL ( $\mathrm{n}=3$ ). (B) 5-bromodeoxyuridine incorporation assay was performed to determine the proliferation of HPASMCs $(\mathrm{n}=5)$. (C) Immunofluorescence assay was used to detect the expression of Ki67. The red color denotes Ki67 staining by rhodamine, whereas the blue color denotes nucleus staining by DAPI ( $=3$ ). Scale bar $=50 \mu \mathrm{m} .{ }^{*} \mathrm{P}<0.05,{ }^{* *} \mathrm{P}<0.01$ and ${ }^{* * *} \mathrm{P}<0.001$. CON, normoxia control; HYP, hypoxia; hyp+si-NC, cells treated with negative control under hypoxic conditions; hyp+si-ANRIL, cells treated with si-ANRIL under hypoxic conditions; HPASMCS, human pulmonary artery smooth muscle cells; PCNA, proliferating cell nuclear antigen; ANRIL, antisense noncoding RNA in the INK4 locus; DAPI, 4,6-diamidino-2-phenylindole.

results revealed that the expression of ANRIL in hypoxic HPASMCs was significantly downregulated compared with that in normoxic cells $(\mathrm{P}<0.01 ;$ Fig. 1$)$.

ANRIL regulates the viability of HPASMCs. To determine the biological function of ANRIL in HPASMCs under hypoxic conditions, siRNA targeting lncRNA ANRIL were designed to inhibit the expression. The efficiency and specificity of siRNA transfection and the downregulation of endogenous IncRNA ANRIL were confirmed by qPCR (Fig. 2A). Meanwhile, to explore the effects of ANRIL on cell survival ability, the viability of HPASMCs was examined by MTT assay. The result revealed the significantly increased viability of hypoxic HPASMCs compared with those under conditions of normoxia, which was strengthened by si-ANRIL under hypoxic conditions ( $\mathrm{P}<0.05$; Fig. $2 \mathrm{~B})$.

ANRIL regulates the cell cycle progression of HPASMCs. In order to investigate whether hypoxia affected cell cycle progression through the IncRNA ANRIL pathway, flow cytometry was used to detect the number of cells at different cell cycle phases. The results showed that hypoxia increased the percentage of cells in the $\mathrm{G} 2 / \mathrm{M}+\mathrm{S}$ phase and the downregulated ANRIL strengthened the increase in the percentage of cells in the G2/M+S phase under hypoxic conditions (Fig. 3).

In addition, cyclins, proteins that regulate the cell cycle and play important roles in both the S phase and G2/M phase of cell cycle, have been generally considered as markers of cellular proliferation. In order to understand the molecular mechanism by which ANRIL regulates the cell cycle, the expression of cell cycle-associated proteins, Cyclin A, Cyclin $\mathrm{D}$ and Cyclin E, were detected by western blotting. The results revealed that the expression of cyclins in hypoxic HPASMCs was significantly increased compared with normoxia, which was further promoted by ANRIL siRNA ( $\mathrm{P}<0.001$; Fig. 4).

ANRIL affects the proliferation of HPASMCs. Subsequently, the effect of ANRIL on HPASMC proliferation under hypoxic conditions was further investigated. The results of western blotting displayed that the expression of PCNA was significantly elevated under hypoxic conditions contrasted with normoxia and si-ANRIL increased the trend further 
A

CON

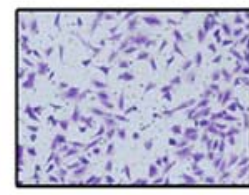

hyp+si-NC

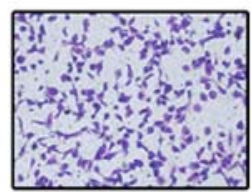

C CON
HYP

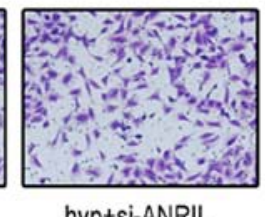

hyp+si-ANRIL

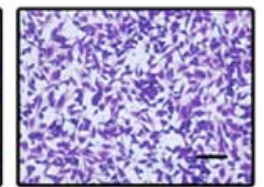

B

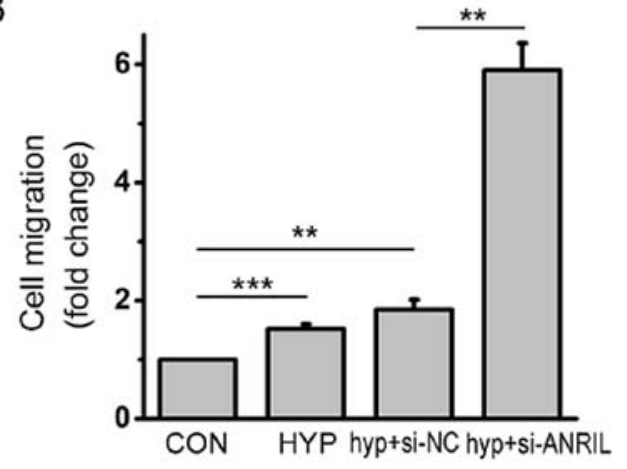

hyp+si-ANRIL

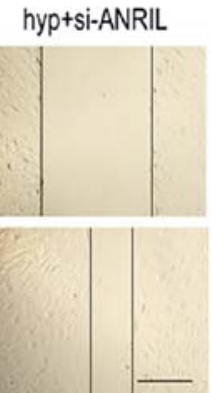

D

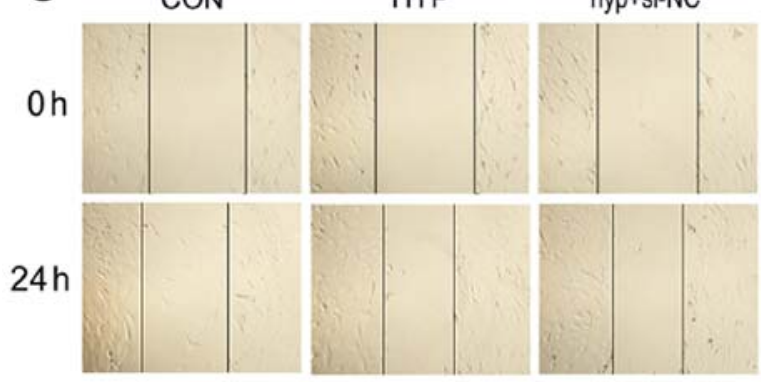
hyp+si-NC

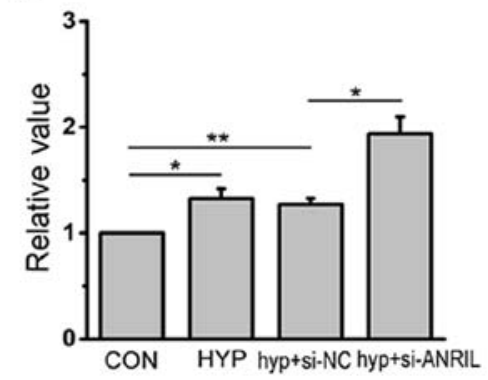

Figure 6. ANRIL induces the migration of HPASMCs under hypoxic conditions. (A) HPASMCs were subjected to a Transwell migration assay. (B) Quantification of the data from the Transwell assay. (C) Scratch-wound assay of HPASMCs after treatment with si-NC or si-lncRNA ANRIL; (D) Quantification of the data from the scratch-wound assay. Scale bar $=100 \mu \mathrm{m}(\mathrm{n}=3)$. ${ }^{*} \mathrm{P}<0.05,{ }^{* * *} \mathrm{P}<0.01$ and ${ }^{* * * *} \mathrm{P}<0.001$. CON, normoxia control; HYP, hypoxia; hyp+si-NC, cells treated with negative control under hypoxic conditions; hyp+si-ANRIL, cells treated with si-ANRIL under hypoxic conditions; HPASMCS, human pulmonary artery smooth muscle cells; ANRIL, antisense noncoding RNA in the INK4 locus.

$(\mathrm{P}<0.05$; Fig. 5A). To evaluate the number of cells that were actively synthesizing DNA, the BrdU incorporation assay was carried out. The present results revealed that the ratio of proliferating cells in HPASMCs under hypoxic conditions was significantly increased compared with under normoxic conditions $(\mathrm{P}<0.001)$, which was enhanced by si-ANRIL (Fig. 5B). The Ki67 protein is a cell proliferation marker that is closely related to cell proliferation and the cell cycle. The result of immunofluorescence assay showed that the expression of Ki67 increased in hypoxia compared with under normal conditions. When the expression of ANRIL was downregulated by siRNA, Ki67 fluorescence staining was found to be increased (Fig. 5C). Consistently, these results demonstrated that the proportion of proliferating cells with a low ANRIL content was higher.

ANRIL promotes migration of hypoxic HPASMCs. The effects of ANRIL on cell migration was examined by Transwell migration assay and scratch-wound assay. As shown in Fig. 6A, the number of migrated cells under hypoxic conditions was more than that under normoxia. Downregulated ANRIL significantly increased the cell migration according to the Transwell migration assay $(\mathrm{P}<0.01$; Fig. 6B). The scratch-wound assay results showed that the migration distance of hypoxic HPASMCs was longer than that in normoxia, which was strengthened by ANRIL siRNA under hypoxic condition in Fig. 6C. Histograms showing the results of the Transwell migration and scratch-wound assays are shown Fig. 6B and D, respectively. Taken together, these data indicated that the downregulation of ANRIL induced by hypoxia enhanced the capacity of migration in HPASMCs.

\section{Discussion}

Previous studies have indicated that, in numerous diseases, lncRNA ANRIL regulates cell proliferation, migration and cell cycle progression including cardiovascular diseases, tumors and diabetes $(18,19)$. In this study, it was found that the expression of ANRIL was downregulated in hypoxic HPASMCs. Hypoxia accelerated the cell cycle progression and induced cell proliferation of HPASMCs through the downregulation of the lncRNA ANRIL pathway. Moreover, downregulated ANRIL in the hypoxic HPASMCs promoted cell migration. Therefore, solid evidence demonstrated that ANRIL is a critical regulator in HPASMCs induced by hypoxia.

It is well established that hypoxia is the main cause of PAH. Recent reports have highlighted the relationship between IncRNAs and hypoxia in PAH. The downregulated lncRNA, maternally expressed 3 , in hypoxic PASMCs was shown to trigger cell proliferation and migration via the p53 signaling pathway (1). The lncRNA Hoxa cluster antisense RNA 3 (Hoxaas3) was upregulated in both pulmonary vessels in hypoxic mice and PASMCs under hypoxic conditions, while a high expression level of Hoxaas 3 was associated with cell proliferation and modulated cell cycle distribution by upregulating Homeobox a3 at the mRNA and protein levels (20). The results from the present study showed that the decrease in the expression level of ANRIL when downregulated in hypoxic HPASMCs was twice that which occurred under normoxic conditions by qPCR, indicating that ANRIL was able to regulate hypoxic PAH.

The proliferation of PASMCs is one of the main causes of medial hypertrophy of PVR in PAH (4). A previous study 
showed that in epithelial ovarian cancer, ANRIL promoted proliferation and cell cycle progression (21). The present study demonstrated that si-ANRIL may accelerate cell cycle progression by increasing the percentage of cells in the $\mathrm{G} 2 / \mathrm{M}+\mathrm{S}$ phase and also elevated the expression level of cyclins under hypoxic conditions. Downregulated ANRIL promoted the expression of PCNA in HPASMCs and increased the expression of Ki-67 under hypoxia. The above results indicated that ANRIL could regulate the cell cycle progression and the proliferation of HPASMCs.

A recently published study reported that the expression of ANRIL in the pancreatic cancer cells was higher than that in normal pancreatic duct epithelial cells and after ANRIL is silenced, the migratory and invasive abilities of the cells were decreased (22). In the present study, Transwell and scratch-wound assays were used to examine the effects of ANRIL on cell migration. The results showed that downregulated ANRIL increased the migration of hypoxic HPASMCs. These data indicated that the downregulation of ANRIL could enhance the capacity of migration in HPASMCs.

At present, the importance of cell biology and the role of ANRIL in the pathogenesis of various diseases have been major topics for study. To the best of our knowledge, this is the first study that has reported on the involvement of ANRIL in PAH, however, there remain several limitations that need to be resolved in future studies. Although the downregulation of ANRIL was detected in HPASMCs, it is still unclear how the decrease of ANRIL is caused by hypoxia. In addition, it is worth noting that one lncRNA may target multiple microRNAs and exert different effects. Whether ANRIL can effectively function as a competing endogenous RNA by binding with microRNAs remains to be further studied. Further experiments will be performed in the future to explore the role of ANRIL in PAH.

In conclusion, the present study has demonstrated that the expression of IncRNA ANRIL in HPASMCs of PAH was reduced. The decreased expression of IncRNA ANRIL contributed to the proliferation and migration of HPASMCs in the pathogenesis of PAH. Therefore, the authors' proposal is that ANRIL-mediated PASMCs are involved in the pathogenesis of vascular remodeling in PAH. These findings provide a novel potential target for the treatment of PAH and may help to improve the therapeutic efforts in the future.

\section{Acknowledgements}

The authors would like to thank Professor Daling Zhu, Dr Ying Liu and Dr Hongyue Zhang (Harbin Medical University) for lending their expertise for the studies.

\section{Funding}

The present study was supported by The Key Project of Natural Science Foundation of Heilongjiang province, project no. 2D201416.

\section{Availability of data and materials}

The datasets used and/or analyzed during the current study are available from the corresponding author on reasonable request.

\section{Authors' contributions}

SW, CZ and XZ made substantial contributions towards the conception and design of the experiments. SW completed all the studies and aggregated the figures and discussed the results. SW and CZ drafted the manuscript and critically revised and added important intellectual content.

\section{Ethics approval and consent to participate}

Not applicable.

\section{Patient consent for publication}

Not applicable.

\section{Competing interests}

The authors declare that they have no competing interests.

\section{References}

1. Sun Z, Nie X, Sun S, Dong S, Yuan C, Li Y, Xiao B, Jie D and Liu Y: Long non-coding RNA MEG3 downregulation triggers human pulmonary artery smooth muscle cell proliferation and migration via the p53 signaling pathway. Cell Physiol Biochem 42: 2569-2581, 2017.

2. Stenmark KR, Fagan KA and Frid MG: Hypoxia-induced pulmonary vascular remodeling: Cellular and molecular mechanisms. Circ Res 99: 675-691, 2006.

3. Howell K, Preston RJ and McLoughlin P: Chronic hypoxia causes angiogenesis in addition to remodelling in the adult rat pulmonary circulation. J Physiol 547: 133-145, 2003.

4. Pietra GG, Capron F, Stewart S, Leone O, Humbert M, Robbins IM, Reid LM and Tuder RM: Pathologic assessment of vasculopathies in pulmonary hypertension. J Am Coll Cardiol 43 (Suppl S): 25S-32S, 2004.

5. Harries LW: Long non-coding RNAs and human disease. Biochem Soc Trans 40: 902-906, 2012.

6. Park JY, Lee JE, Park JB, Yoo H, Lee SH and Kim JH: Roles of long non-coding RNAs on tumorigenesis and glioma development. Brain Tumor Res Treat 2: 1-6, 2014.

7. Li T, Mo X, Fu L, Xiao B and Guo J: Molecular mechanisms of long noncoding RNAs on gastric cancer. Oncotarget 7: 8601-8612, 2016.

8. Roberts R: Genetics of coronary artery disease: An update. Methodist Debakey Cardiovasc J 10: 7-12, 2014.

9. Pasmant E, Laurendeau I, Héron D, Vidaud M, Vidaud D and Bièche I: Characterization of a germ-line deletion, including the entire INK4/ARF locus, in a melanoma-neural system tumor family: Identification of ANRIL, an antisense noncoding RNA whose expression coclusters with ARF. Cancer Res 67: 3963-3969, 2007.

10. Tano $\mathrm{K}$ and Akimitsu N: Long non-coding RNAs in cancer progression. Front Genet 3: 219, 2012.

11. Schaefer AS, Bochenek G, Manke T, Nothnagel M, Graetz C, Thien A, Jockel-Schneider Y, Harks I, Staufenbiel I, Wijmenga C, et al: Validation of reported genetic risk factors for periodontitis in a large-scale replication study. J Clin Periodontol 40: 563-572, 2013.

12. Masharawi YM, Kjaer P, Bendix T, Manniche C, May H, Mirovsky Y, Anekshtein Y, Jensen TS and Hershkovitz I: Lumbar facet and interfacet shape variation during growth in children from the general population: A three-year follow-up MRI study. Spine (Phila Pa 1976) 34: 408-412, 2009.

13. Congrains A, Kamide K, Katsuya T, Yasuda O, Oguro R, Yamamoto K, Ohishi M and Rakugi H: CVD-associated non-coding RNA, ANRIL, modulates expression of atherogenic pathways in VSMC. Biochem Biophys Res Commun 419: 612-616, 2012.

14. Zhou X, Han X, Wittfeldt A, Sun J, Liu C, Wang X, Gan LM, Cao $\mathrm{H}$ and Liang $\mathrm{Z}$ : Long non-coding RNA ANRIL regulates inflammatory responses as a novel component of NF- $\mathrm{KB}$ pathway. RNA Biol 13: 98-108, 2016. 
15. Livak KJ and Schmittgen TD: Analysis of relative gene expression data using real-time quantitative PCR and the 2(-Delta Delta C(T)) method. Methods 25: 402-408, 2001.

16. Yu X, Li T, Liu X, Yu H, Hao Z, Chen Y, Zhang C, Liu Y, Li Q, Mao $M$ and Zhu D: Modulation of pulmonary vascular remodeling in hypoxia: Role of 15-LOX-2/15-HETE-MAPKs Pathway. Cell Physiol Biochem 35: 2079-2097, 2015.

17. Siegel R, Ma J, Zou Z and Jemal A: Cancer statistics, 2014. CA Cancer J Clin 64: 9-29, 2014.

18. Aguilo F, Di Cecilia S and Walsh MJ: Long Non-coding RNA ANRIL and Polycomb in human cancers and cardiovascular disease. Curr Top Microbiol Immunol 394: 29-39, 2016.

19. Zhang B, Wang D, Ji TF, Shi L and Yu JL: Overexpression of IncRNA ANRIL up-regulates VEGF expression and promotes angiogenesis of diabetes mellitus combined with cerebral infarction by activating NF- $\kappa \mathrm{B}$ signaling pathway in a rat model. Oncotarget 8: 17347-17359, 2017.

20. Zhang H, Liu Y, Yan L, Wang S, Zhang M, Ma C, Zheng X, Chen $\mathrm{H}$ and Zhu D: Long noncoding RNA Hoxaas 3 contributes to hypoxia-induced pulmonary artery smooth muscle cell proliferation. Cardiovasc Res 115: 647-657, 2018.
21. Qiu JJ, Wang Y, Liu YL, Zhang Y, Ding JX and Hua KQ: The long non-coding RNA ANRIL promotes proliferation and cell cycle progression and inhibits apoptosis and senescence in epithelial ovarian cancer. Oncotarget 7: 32478-32492, 2016.

22. Chen S, Zhang JQ, Chen JZ, Chen HX, Qiu FN, Yan ML, Chen YL, Peng CH, Tian YF and Wang YD: The over expression of long non-coding RNA ANRIL promotes epithelial-mesenchymal transition by activating the ATM-E2F1 signaling pathway in pancreatic cancer: An in vivo and in vitro study. Int J Biol Macromol 102: 718-728, 2017.

cc (i) (9) This work is licensed under a Creative Commons Attribution-NonCommercial-NoDerivatives 4.0 International (CC BY-NC-ND 4.0) License. 